Bio-grafía Escritos sobre la Biologia y su Enseñanza.

Edición Extra-Ordinaria. ISSN 2027-1034 P. p245 - 253

Memorias del VII Encuentro Nacional de Experiencias en la Enseñanza de la Biologia y la Educación Ambiental y II Congreso Nacional de Investigación en la Enseñanza de la Biología

\title{
APRENDIZAJE SIGNIFICATIVO DEL CONCEPTO REPRODUCCIÓN EN ESTUDIANTES DE GRADO QUINTO DE PRIMARIA
}

\section{SIGNIFICANT LEARNING CONCEPT STUDENTS REPRODUCTION IN FIFTH GRADE OF ELEMENTARY}

\section{María Angélica Rincón ${ }^{1}$}

\section{Paola Andrea Rodríguez ${ }^{2}$}

\section{Resumen}

El diseño de la unidad didáctica caminando ando, por la reproducción, se convierte en una propuesta pedagógica de formación de docentes investigadores, en esta, se desarrolló el concepto de reproducción, dirigida a estudiantes de grado quinto de primaria de la Institución Educativa Distrital Colegio Externado Nacional Camilo Torres, bajo el enfoque de aprendizaje significativo y por medio del paradigma investigativo socio crítico, que permitió la comprensión y aprendizaje del concepto, por parte de los estudiantes con ayuda de las estrategias Pre-instruccionales, Co-instruccionales y post-instruccionales, obteniendo como resultados una transformación en las ideas previas de los estudiantes pasando de una mirada macro a una mirada micro comprendiendo y asimilando el concepto de reproducción y la finalidad de este como proceso por el cual se prolonga una especie.

Palabras Clave Aprendizaje significativo, didáctica, docente, reproducción, estrategias.

\section{Abstrac}

The design of the teaching unit'm walking through the reproduction, becomes a pedagogical teacher education researchers, in this, developed the concept of reproduction, aimed at fifth grade students of School Elementary School District National externship Camilo Torres, under the approach of learning through meaningful and critical partner research paradigm, which allowed the understanding and learning of the concept, by using students Pre-instructional strategies, Co-instructional and post-instructional, results obtained as a transformation in the students' previous ideas moving from a macro look at a micro look understanding and assimilating the concept of reproduction and the purpose of this as a process by which extends a species.

\footnotetext{
1 Universidad Distrital Francisco José de Caldas, Proyecto curricular Licenciatura en biología, Aula Experimental y Recursos Educativos. mangelk89@hotmail.com

2 Universidad Distrital Francisco José de Caldas, Proyecto curricular Licenciatura en biología, Aula Experimental y Recursos Educativos. andreita920129@gmail.com
} 
Bio-grafía Escritos sobre La Biologia y su Enseñanza.

Edición Extra-Ordinaria. ISSN 2027-1034 P. p245 - 253

Memorias del VII Encuentro Nacional de Experiencias en la Enseñanza de la

Biología y la Educación Ambiental y II Congreso Nacional de Investigación en

la Enseñanza de la Biología

Keywords Meaningful learning, teaching, teacher, reproduction, strategies.

\section{Introducción}

En la actualidad la formación de los docentes en el área investigativa ha cobrado vital importancia, debido a que el maestro investigador es aquel que indaga, crea y reflexiona sobre las problemáticas de la enseñanza aprendizaje, la formación de los docentes-investigadores no puede realizarse sólo en el aula, recibiendo clases, implica una actitud de búsqueda permanente y un interés por la transformación de las situaciones que se viven en las escuelas en el sentido de la emancipación y el como la práctica se convierte en un espacio reflexivo y crítico del quehacer docente desde una mirada personal (Carr 1943, citado por Méndez 2007).

La unidad didáctica se encuentra definida por tres etapas, la primera una caracterización esta se enfocó en tres pilares de la comunidad educativa: la institución mediante el conocimiento de su P.E.I., y el enfoque educativo de la institución, el docente del grado quinto de primaria de la Institución Educativa Distrital Colegio Externado Nacional Camilo Torres, y caracterización del estudiante.

En segundo lugar tenemos el diseño de la unidad didáctica, interrelacionando lo obtenido en la caracterización, se elaboró acorde a la realidad escolar de la Institución, para el grado quinto de primaria, la unidad fue trabajada bajo el enfoque de aprendizaje significativo planteado por Ausubel 1973 (citado por Ausubel 1978) como las ideas, conceptos y proposiciones específicamente relevantes e inclusivas de las estructura cognoscitiva del alumno, tiene como propósito relacionar los nuevos conceptos a partir de las ideas previas del estudiante. El aprendizaje significativo se produce cuando se relaciona información nueva con algún concepto inclusor ya existente en la estructura cognitiva del individuo que resulte relevante para el nuevo material que se intenta aprender, Pozo 2006 (citado por Garzón \& Gamboa 2010). Por medio del enfoque, lo que se plantea es la construcción y comprensión por parte de los estudiantes sobre el concepto de reproducción y la finalidad de esta, dando las ideas del concepto en orden lógico y una secuencia de lo micro a lo macro.

El conocimiento que tienen los estudiantes lo construyen a partir de su interacción con el entorno físico y social, para explicar la construcción de dicho conocimiento, es necesario retomar la teoría Piagetiana, en la cual se afirma que la mente humana pasa por dos procesos en esta construcción: la asimilación y la acomodación. Los niños y niñas tienen concepciones acerca de la reproducción, las cuales han construido en todos los entornos en los cuales interactúan, a partir de las explicaciones que las personas dan a este fenómeno (Hernández et al 2010), para la construcción de un concepto biológico sobre la reproducción por parte de los estudiantes, es necesario la revisión de textos, ilustraciones, mapas 
Bio-grafía Escritos sobre La Biologia y su Enseñanza.

Edición Extra-Ordinaria. ISSN 2027-1034 P. p245 - 253

Memorias del VII Encuentro Nacional de Experiencias en la Enseñanza de la

Biología y la Educación Ambiental y II Congreso Nacional de Investigación en

la Enseñanza de la Biología

conceptuales y gráficos que los ayuden a la interpretación de dicho concepto y cautive la atención de estos para que haya un aprendizaje significativo.

\section{Metodología}

La experiencia de la implementación de la unidad didáctica se desarrolló en la Institución Educativa Distrital Colegio Externado Nacional Camilo Torres, en el grado quinto de primaria, el grupo estaba conformado por 44 estudiantes, en un rango de edad de 9-13 años, mediante la etapa de caracterización se conoció a los estudiantes y se reconoció las ideas previas que tenían frente al concepto de reproducción y cuál era su motivación para asistir al colegio.

El paradigma investigativo socio-crítico de acuerdo con Arnal (1992) tiene como objetivo promover las transformaciones sociales, dando respuestas a problemas específicos presentes en el seno de las comunidades, pero con la participación de sus miembros, por tanto el conocimiento se construye con la participación de toda la comunidad educativa en este caso, educandos, docente titular y docenteestudiante.

Entre las características más importantes del del paradigma socio-crítico aplicado al ámbito de la educación se encuentran: (a) la adopción de una visión global y dialéctica de la realidad educativa; (b) la aceptación compartida de una visión democrática del conocimiento así como de los procesos implicados en su elaboración; y (c) la asunción de una visión particular de la teoría del conocimiento y de sus relaciones con la realidad y con la práctica. (Alvarado, L. \& García, M. 2008), para la aplicación de este paradigma investigativo en la práctica se utilizaron los siguientes instrumentos la observación participante por parte del docente-estudiante, con una continua reflexión, análisis y autocrítica de la construcción de la formación docente, el concepto sobre reproducción y las implicaciones sociales de la práctica, orientadas hacia las necesidades educativas y sociales de los estudiantes; y el diario de campo como estrategia para sistematizar las experiencias en el aula de clase, para posteriormente realizar una análisis e interpretación de las vivencias.

Con los resultados arrojados en la etapa de caracterización se diseñó la unidad, la cual partió desde las ideas que tienen los estudiantes, se realizó un cronograma de actividades, en el cual se planteaban cada una de las sesiones de clase, en concordancia con el enfoque escogido, el concepto se desarrolló de forma jerarquizada, cada clase constaba de una hora con treinta minutos de ejecución, sin embrago es de gran importancia mencionar que las actividades se realizaban teniendo en cuenta las ideas previas que surgían por parte de los estudiantes en la sesión de clase anterior: 
Bio-grafía Escritos sobre La Biologia y su Enseñanza.

Edición Extra-Ordinaria. ISSN 2027-1034 P. p245 - 253

Memorias del VII Encuentro Nacional de Experiencias en la Enseñanza de la Biología y la Educación Ambiental y II Congreso Nacional de Investigación en la Enseñanza de la Biología

\begin{tabular}{|l|l|l|}
\hline \multicolumn{1}{|c|}{ CLASE } & \multicolumn{1}{|c|}{ OBJETIVO } & ACTIVIDAD \\
\hline $\begin{array}{l}\text { 1. Introducción a la } \\
\text { reproducción }\end{array}$ & $\begin{array}{l}\text { Acercar e introducir al estudiante al desarrollo } \\
\text { histórico del concepto, y la finalidad global que } \\
\text { tiene para los organismos }\end{array}$ & $\begin{array}{l}\text { Reproducción } \\
\text { el comic }\end{array}$ \\
\hline $\begin{array}{l}\text { 2. Reproducción } \\
\text { asexual }\end{array}$ & $\begin{array}{l}\text { Relacionar a los estudiantes con el concepto de } \\
\text { reproducción asexual y los tipos que se } \\
\text { presentan }\end{array}$ & $\begin{array}{l}\text { Uniendo y } \\
\text { aprendiendo }\end{array}$ \\
\hline $\begin{array}{l}\text { 3.Reproducción } \\
\text { asexual }\end{array}$ & Explicar los tipos de reproducción en plantas & $\begin{array}{l}\text { Folleto de } \\
\text { reproducción } \\
\text { asexual }\end{array}$ \\
\hline $\begin{array}{l}\text { 4.Introducción a la } \\
\text { reproducción sexual }\end{array}$ & $\begin{array}{l}\text { Reconocer la reproducción sexual como un } \\
\text { mecanismo que produce variabilidad en las } \\
\text { especies }\end{array}$ & $\begin{array}{l}\text { Elaboración } \\
\text { de un mapa } \\
\text { conceptual }\end{array}$ \\
\hline $\begin{array}{l}\text { 5.Reproducción } \\
\text { sexual }\end{array}$ & $\begin{array}{l}\text { Relacionar al estudiante con los tipos de cortejos } \\
\text { y la fecundación interna y externa }\end{array}$ & $\begin{array}{l}\text { Video "cortejo } \\
\text { de animales } \\
\text { 2" }\end{array}$ \\
\hline $\begin{array}{l}\text { 6.La reproducción, } \\
\text { tecnología y } \\
\text { sociedad }\end{array}$ & $\begin{array}{l}\text { Mostrar a los estudiantes el papel que juega la } \\
\text { biotecnología y la reproducción hoy en día. }\end{array}$ & $\begin{array}{l}\text { Los bebé } \\
\text { probeta }\end{array}$ \\
\hline
\end{tabular}

Tabla 1. Sesiones de clases realizadas para el desarrollo de la unidad didáctica

Para evaluar la asimilación del concepto de reproducción, se trabajó con lo propuesto por Barriga, F \& Hernández, G. 1999 ya que permite utilizar una serie de estrategias que pueden incluirse en el manejo de un tema en específico, la cual consiente en evaluar en 3 diferentes momentos como se encuentra la temática desarrollada por el docente y la asimilación que esta presentado en ellos, estas estrategias son las pre-instruccionales preparan y alertan al estudiante en relación a qué y cómo va aprender, es un mecanismo en el cual se conocen las ideas previas de los estudiantes; Las co-instruccionales apoyan los contenidos curriculares durante el proceso mismo de enseñanza; y finalmente las postinstruccionales permiten al alumno formar una visión sintética, integradora e incluso crítica sobe el concepto que se aprendió.

\section{Resultados Y Análisis}

Los investigadores han intentado determinar de dónde se origina estas ideas previas y al parecer están determinadas por esquemas conceptuales que están ampliamente extendidos en todas las culturas, por tanto las ideas previas son una construcción cultural y social que se desarrolló de acuerdo a interacción que tiene 
Bio-grafia Escritos sobre la Biologia y su Enseñanza.

Edición Extra-Ordinaria. ISSN 2027-1034 P. p245 - 253

Memorias del VII Encuentro Nacional de Experiencias en la Enseñanza de la

Biología y la Educación Ambiental y II Congreso Nacional de Investigación en

la Enseñanza de la Biología

el individuo con el ambiente donde se interactúa. Según Duit, 1991; Pozo, Sanz, Gómez y Limón, 1991 algunas de las ideas previas sobre fenómenos científicos tienen su origen en el uso de analogías defectuosas en el propio medio, lo cual indica que en ocasiones como docentes por el mal uso de las analogías se crean confusiones en donde el sujeto cree que el objeto de analogía es el fenómeno que se está estudiando.

Para desarrollar la estrategia pre-instruccional, se utilizaron las ideas previas que tenían los estudiantes sobre reproducción las cuales se obtuvieron del proceso de caracterización, algunos de los resultados presentados en los estudiantes muestran que para ellos el concepto de reproducción es el proceso por el cual se trae un ser al mundo, el estudiante tiene una idea de reproducción bio-sexista, donde la reproducción es un proceso exclusivo de relaciones sexuales en donde se tiene que presentar un contacto físico entre los organismos y que además entre ellos debe haber una diferenciación sexual como lo hombre y mujer o macho y hembra. En muchos de los esquemas presentados por los estudiantes se encontraba que para ellos la reproducción era el concepto básico de vida en donde los organismos nacen, crecen, se reproducen y mueren, como menciona Pintó, Aliberas y Gómez, (1996) que aunque las ideas espontáneas son construcciones personales y propias de cada sujeto, existen muchas más semejanzas que diferencias entre ellas, lo que ha permitido identificar algunas representaciones comunes en alumnos de países y sistemas educativos, en los estudiantes del grado quinto de primaria la idea que se tenía de reproducción era el concepto de vida debido a que la gran mayoría de los estudiantes, han escuchado la palabra reproducción en la escuela, por parte de las maestras pero muchos manifestaban no conocer el significado de dicha palabra.

En cuanto a la segunda etapa de asimilación del concepto de reproducción asexual y la compresión del concepto etapa co-instruccional, después de haber realizado la introducción, y de manejar el contenido de manera jerarquizada, se realiza en la clase número 3 la elaboración de un folleto en donde los niños sean libres de plasmar lo que han comprendido acerca del concepto de reproducción.

\footnotetext{
" yo entendí que la Hydra viridis presenta reproducción asexual osea que se reproduce ella sola y también aprendí que la reproducción asexual es de los animales que no necesitan de una pareja para crear un nuevo ser o un nuevo organismo y que hay varias plantas que presentan reproducción asexual y se llama esporulación osea que se reproducen solas por un medio de esporas y que unos hongos también se reproducen por medio de esporas y hay unas esporas que caen al suelo y nacen pero no todas porque hay depredadores que se las comen y la mata de fresa saca un estolón para darle vida a otra planta."
} 
Bio-grafía Escritos sobre La Biologia y su Enseñanza.

Edición Extra-Ordinaria. ISSN 2027-1034 P. p245 - 253

Memorias del VII Encuentro Nacional de Experiencias en la Enseñanza de la

Biología y la Educación Ambiental y II Congreso Nacional de Investigación en la Enseñanza de la Biología

Parrafo 1. Escrito de un folleto realizado sobre el concepto de reproducción y reproducción asexual

Como podemos observar el párrafo presentado No.1, evidencia un avance en la asimilación del concepto de reproducción, los estudiantes realizan una modificación de sus ideas previas de reproducción más allá del concepto de vida, que era la idea en común que presentaban los estudiantes, además como menciona Ausubel (1983) (citado en Ausubel 1986), en esta etapa se está presentando el aprendizaje subordinado, en donde los estudiantes toman las ideas de mayor abstracción y las relacionan con las ideas establecidas. Se logró que los estudiantes cambiaran las ideas bio-sexistas, en donde no se necesitan dos progenitores y el acto sexual para que se presente reproducción.

En cuanto a las estrategias post instruccionales se realizaron en la sesión número seis, la actividad consistió en la elaboración de un mapa conceptual sobre el concepto de reproducción y un esquema o dibujo donde es incluyera todo lo tratado en clase sobre el concepto, los resultados obtenidos fueron los siguientes:

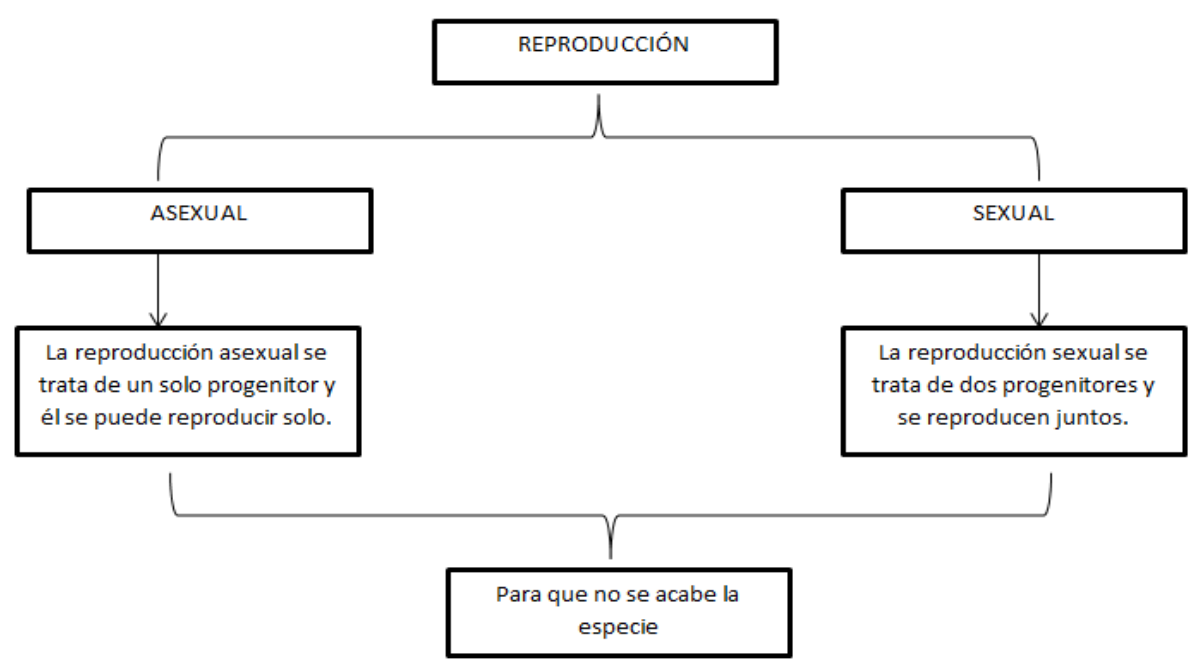

Figura No. 1 Estrategia post- instruccionales, mapa conceptual estado de asimilación del concepto de reproducción 
Bio-grafía Escritos sobre La Biologia y su Enseñanza.

Edición Extra-Ordinaria. ISSN 2027-1034 P. p245 - 253

Memorias del VII Encuentro Nacional de Experiencias en la Enseñanza de la Biología y la Educación Ambiental y II Congreso Nacional de Investigación en la Enseñanza de la Biología

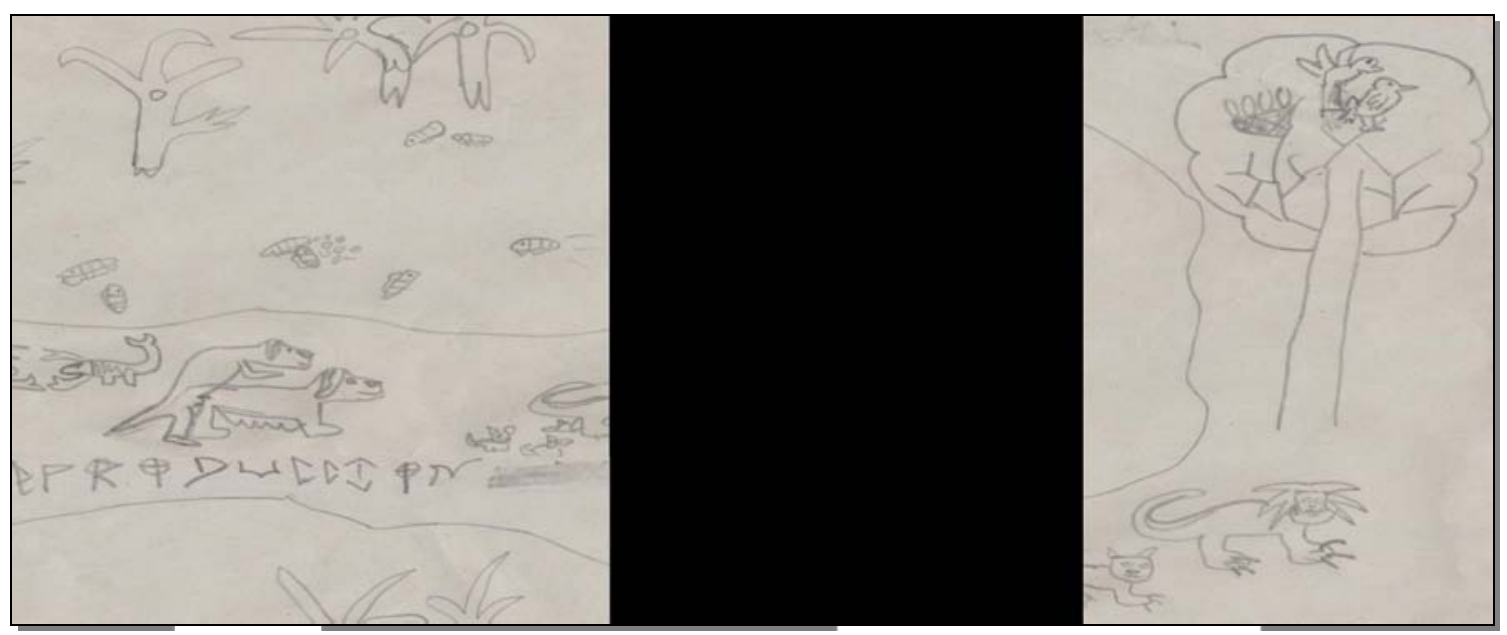

Figura No. 2 Estrategia post- instruccionales esquema estado de asimilación del concepto de reproducción

Esta actividad se realiza después del video presentado "Cortejo de animales 2", el cual permitió incentivar la motivación intrínseca en los estudiantes, como mencionan Ausubel, Novak, Hanesian (1978) la motivación es tanto un efecto como una causa del aprendizaje, en donde conviene elevar al máximo el impulso cognoscitivo.

Para Symington y Novak (1982) (Citado en Gonzales F. 1992), la elaboración de un mapa ayuda a los profesores a ver la naturaleza conceptual y del conocimiento y su relación con la construcción humana, además por medio de estos se pueden identificar los subsunsores que son aspectos relevantes de la estructura cognitiva y la organización secuencial que se le está dando al conocimiento, como podemos observar en la figura No. 1, el estudiante realiza una organización secuencial en el concepto de reproducción, en cuanto al ¿para que se presenta la reproducción?, los estudiantes mencionan: para que no se acabe la especie, algunos mencionaron para que no se extinga y otros para que se propague, por tanto, el para qué de la reproducción fue asimilado por los estudiantes, también se observó la asimilación de la diferenciación entre reproducción sexual y asexual y desapareció la idea bio-sexista que se tenía frente a la reproducción como acto sexual. En lo correspondiente al dibujo Figura No. 2 el estudiante recrea un ambiente propicio para que se presente la reproducción, en donde relaciona a diversos organismos, manifestando de esta forma que los diferentes tipos de reproducción se presentan en un mismo ecosistema.

\section{Conclusiones}

Los procesos de reflexión y auto -crítica en la práctica docente, permiten la evaluación y creación de diversas estrategias que permitan problematizarse en el 
Bio-grafía Escritos sobre la Biologia y su Enseñanza.

Edición Extra-Ordinaria. ISSN 2027-1034 P. p245 - 253

Memorias del VII Encuentro Nacional de Experiencias en la Enseñanza de la

Biología y la Educación Ambiental y II Congreso Nacional de Investigación en la Enseñanza de la Biología

proceso de enseñanza-aprendizaje acorde con las problemáticas presentadas en el aula de clase.

Para la comprensión y aprendizaje del concepto las ideas previas de los estudiantes juegan un papel importante, el maestro debe partir de dichas ideas para la explicación y transformación del concepto, permitiéndole a los niños hacer una conexión entre la información que se está aprendiendo y la que se presentaba.

En cuanto a la asimilación del concepto por parte de los estudiantes, se observó un cambio en la estructura cognoscitiva que se evidencio con la vinculación y el fortalecimiento de los conceptos expuestos en clase, lo cual se pudo evidenciar por la vinculación de la información presentada y la manera secuencial como esta se estaba organizando en la estructura cognoscitiva del estudiante, lo cual se expresó por medio de la elaboración de un mapa conceptual.

\section{Referencias Bibliográficas}

Alvarado, L. \& García, M. (2008) Características más relevantes del paradigma socio-crítico: su aplicación en investigaciones de educación ambiental y de enseñanza de las ciencias realizadas en el Doctorado de Educación del Instituto Pedagógico de Caracas Sapiens. Revistas científicas de América Latina. Vol. 9, Núm. 2, diciembre, 2008, pp. 187-202 Universidad Pedagógica Experimental Libertador Venezuela

Arnal, J. (1992). Investigación educativa. Fundamentos y metodología. Barcelona (España): Labor

Ausubel, D.P., Novak, J.D. \& Hanesian, H. (1986). Educational psychology: a cognitive view. New York: Holt, Rinehart and Winston. Publicado en portugués por la Editora Interamericana, Rio de Janeiro, 1980. En español por Editorial Trillas, México, 1981. Reimpreso en inglés por Werbel \& Peck, New York.

Barriga, F \& Hernández, G. (1999). Estrategias de enseñanza para la promoción de aprendizajes significativos. McGraw Hill. México.

Fanjul, M \& Hiriat, M. (2008), Biología Funcional de los Animales I vol. 1. México D. C.

Fonseca, G. (2009). La formación de profesores de biologia a través del diseño, implementación y sistematización de unidades didácticas. Enseñanza de las Ciencias, Número Extra VIII Congreso Internacional sobre Investigación en Didáctica de las Ciencias, Barcelona, pp. 2300-2303 
Bio-grafía Escritos sobre la Biología y su Enseñanza.

Edición Extra-Ordinaria. ISSN 2027-1034 P.p245 - 253

Memorias del VII Encuentro Nacional de Experiencias en la Enseñanza de la

Biología y la Educación Ambiental y II Congreso Nacional de Investigación en la Enseñanza de la Biología

Garzon N., Gamboa A., (2010), La enseñanza de la célula desde un aprendizaje significativo para estudiantes de quinto de primaria. Asociación Colombiana para la investigación en Ciencias Y Tecnología EDUC y T, Memorias, II congreso Nacional de investigación en educación en ciencias y tecnología.

Gonzales F. (1992). Los mapas conceptuales de J. Novak como instrumentos para la investigación en didáctica de las ciencias experimentales. Departamento de Filosofía y Metodología de las Ciencias. Universidad Pública de Navarra. Pamplona.

Hernández M., Pardo A., Tovar S., (2010), Comprensión de las concepciones sobre reproducción sexual de los animales vertebrados que tienen los niños y niñas de quinto grado de primaria. Universidad Tecnológica de Pereira, Pereira Colombia.

Méndez A. Méndez S., (2007) El docente investigador en educación, Universidad de ciencias y artes en Chiapas, México.

Pintó, R., Aliberas, J. \& Gómez, R. (1996). Tres enfoques de la investigación sobre concepciones alternativas. Enseñanza de las Ciencias, 14, pp. 221-232

Rosenzweig M., Breedlove S., Watson N., (2005) Psicobiologia una introduccion a la neurociencia conductual, cognitiva y clínica, Ed. Ariel S.A. España.

Villee Claude A., Salomon E., Martin D., Berg I., Palacios R., (1998) Biología, Tercera edición, México D.F.

Vygotski, I. S. (1981) Pensamiento y lenguaje. Teoría del desarrollo cultural de las funciones psíquicas. Buenos Aires: La Pléyade 